\title{
Lactitol, a new hydrogenated lactose derivative: intestinal absorption and laxative threshold in normal human subjects
}

\author{
By DHARMARAJ H. PATIL, GEORGE K. GRIMBLE AND \\ DAVID B. A. SILK \\ Department of Gastroenterology \& Nutrition, Central Middlesex Hospital, \\ Acton Lane, London NW10 $7 N S$
}

(Received 18 December 1985 - Accepted 15 September 1986)

\begin{abstract}
1. In the first part of the study, the absorption of lactitol, a new disaccharide analogue of lactose, was studied using an in vivo jejunal perfusion technique in man. Intestinal uptake of lactitol from isotonic solutions containing $10,30,60$, and $100 \mathrm{mmol}$ lactitol $/ 1$ was insignificant.

2. In the second part of the study the laxative threshold of lactitol was determined and compared with that of sorbitol in a double-blind, randomized, cross-over study on twenty-one normal subjects. Laxative threshold was considered to be either the maximum dose tolerated without unacceptable diarrhoea or gastrointestinal side effects, or when the maximum dose in the study was reached. Increasing amounts of lactitol, sorbitol or placebo were administered in two divided doses each day until subjects developed diarrhoea or severe gastrointestinal side effects. The laxative threshold of lactitol (74 (SE 5) g/d) was similar to that of sorbitol (71 (SE 5) g/d).

3. These findings indicate that lactitol is not absorbed by the human small intestine. Although diarrhoea or other gastrointestinal side effects occurred as the dose was increased, $40 \mathrm{~g}$ lactitol $/ \mathrm{d}$ was well tolerated.
\end{abstract}

Sucrose, which adds bulk and texture to food products, has traditionally been the most widely used sweetening agent in foodstuffs. The major disadvantage is its high energy value, and because of increasing concern over the prevalence of obesity and diabetes mellitus, it is now considered desirable to develop alternative sweetening agents of lower energy value. Sorbitol, a sugar alcohol derived from glucose, is poorly absorbed in man (Wick et al.1951) and is an example of the type of compound that is now being used as a low-energy sweetening agent.

Recently, lactitol (4-O-( $\beta$-galactopyranosyl)-D-sorbitol), a dissacharide analogue of lactose, has been described (van Valthuijsen, 1979). Animal experiments and low-dose oral tolerance tests in normal subjects suggest that lactitol is not absorbed from the mammalian small intestine (Saijonmaa et al. 1978; van Valthuijsen, 1979). As lactitol has a pleasant sweet taste and is less hygroscopic than sucrose (Saijonmaa et al. 1978), this sugar also has potential in the food industry.

In the present study, this potential of lactitol has been investigated in man in two ways. Firstly, small intestinal absorption has been characterized over a range of concentrations using an in vivo steady-state perfusion technique. Secondly, because any non-absorbable sugar will produce an osmotic diarrhoea if consumed in large enough quantities, we have determined its laxative threshold.

\section{METHODS \\ Jejunal perfusion experiments}

Six normal volunteers aged between 20 and 30 years, who gave their informed consent, were intubated with a double-lumen perfusion tube incorporating a proximal occlusive balloon (Sladen \& Dawson, 1970). The tube was allowed to pass until the infusion orifice was distal to the duodeno-jejunal flexure, and the final position was checked radiologically so that the $0.3 \mathrm{~m}$ perfusion segment was positioned in the upper jejunum. The test solutions, 
Table 1. Gastrointestinal symptoms and scoring

\begin{tabular}{ll}
\hline \hline Symptoms & \multicolumn{1}{c}{ Scoring } \\
\hline Flatus & 0 No change \\
Abdominal pain & 1 Barely-noticeable change \\
Bloating & 2 Noticeable, not interfering with activity \\
Borborygmi & 3 Mild interference with activity \\
& 4 Moderate interference with activity \\
& 5 Severe interference with activity \\
\hline
\end{tabular}

maintained at $37^{\circ}$ in a water-bath, were perfused at a rate of $15 \mathrm{ml} / \mathrm{min}$ using a constant-infusion peristaltic pump (MHRE 200; Watson-Marlow Ltd). Four test solutions were perfused in random order. After an equilibration period of $30 \mathrm{~min}$, three $10-\mathrm{min}$ samples were collected from the distal collecting orifice by simple siphonage. The samples were collected into a container chilled in crushed ice and were then stored at $4^{\circ}$ until analysed on the same day.

The test solutions contained lactitol at concentrations of $10,30,60$ and $100 \mathrm{mmol} / \mathrm{l}$, labelled with $1 \mu \mathrm{Ci}\left[1^{14} \mathrm{C}\right]$ lactitol/1 (Amersham International plc, Amersham, Bucks). All solutions were made isotonic with plasma by adding sodium chloride and each contained $4 \mathrm{~g}$ polyethylene glycol (molecular weight 4000 ; $\mathrm{PEG}$ )/ $/$ labelled with $1 \mu \mathrm{Ci}{ }^{3} \mathrm{H}$-labelled PEG/l.

\section{Analysis and calculation of results}

The galactose content of test solutions and intestinal aspirates was analysed using a galactose dehydrogenase (EC I I I 1 48)-based assay (Rommel et al. 1968) in kit form (Boehringer Corporation (London) Ltd, Lewes, Sussex). Preliminary experiments established that lactitol did not interfere with the galactose assay. The amounts of labelled PEG and lactitol were measured by liquid-scintillation counting with a Beckman LS 7500 (Beckman RIIC, High Wycombe, Bucks) using a dual-labelled-isotope counting program. Portions $(1.0 \mathrm{ml})$ of sample were mixed with a $2.5 \mathrm{ml}$ scintillant (Beckman-MP) and quench correction was performed using the $\mathrm{H}$-number method. Absorption of lactitol (expressed as percentage of infused load) was calculated as described previously (Holdsworth \& Dawson, 1964).

\section{Laxative threshold study}

Twenty-one normal subjects (eleven female) aged between 23 and 48 years with mean body-weight $60.4 \mathrm{~kg}$ were entered into a double-blind, cross-over study. Each subject ingested, twice daily, lactitol $(20 \mathrm{~g})$, sorbitol $(10 \mathrm{~g})$ or placebo (glucose $20 \mathrm{~g}$ ) in random order and the daily dose was increased by $5 \mathrm{~g}$ twice daily for $9 \mathrm{~d}$. The sugars were dissolved in $200 \mathrm{ml}$ water and ingested within $0.5 \mathrm{~h}$ after morning and evening meals. There was a minimum $4 \mathrm{~d}$ interval between each of the study periods.

A daily record of gastrointestinal symptoms (flatus, abdominal pain, bloating, borborgymi) was kept by all subjects during each study period and symptoms were scored according to criteria summarized in Table 1 . The administration of test substances was discontinued if there was a total symptom score of $>12 / 24 \mathrm{~h}$ or a single symptom score $>5 / 24 \mathrm{~h}$ or if the subjects developed more than four bowel motions $/ 24 \mathrm{~h}$. For the purpose of the study, the laxative threshold was defined as the dose at which the test substance produced unacceptable levels of side effects or when the maximum dose in the study $(130 \mathrm{~g} / 24 \mathrm{~h})$ was reached. 
Table 2. Percentage disappearance of $\left[{ }^{14} \mathrm{C}\right]$ lactitol during jejunal perfusion experiments in human subjects

(Mean values with their standard errors)

\begin{tabular}{|c|c|c|c|c|c|c|c|c|}
\hline & \multicolumn{8}{|c|}{ Concentrations of lactitol in perfusion solutions (mmol/l) } \\
\hline & \multicolumn{2}{|c|}{10} & \multicolumn{2}{|c|}{20} & \multicolumn{2}{|c|}{60} & \multicolumn{2}{|c|}{100} \\
\hline & Mean & SE & Mean & SE & Mean & $\mathrm{SE}$ & Mean & SE \\
\hline $\begin{array}{c}\text { Disappearance of } \\
{\left[{ }^{14} \mathrm{C}\right] \text { lactitol }(\%)}\end{array}$ & -0.66 & $2 \cdot 11$ & $0 \cdot 11$ & 0.64 & -0.06 & 0.60 & $-1 \cdot 00$ & 0.94 \\
\hline$n$ & \multicolumn{2}{|c|}{5} & \multicolumn{2}{|c|}{4} & \multicolumn{2}{|c|}{5} & \multicolumn{2}{|c|}{5} \\
\hline
\end{tabular}

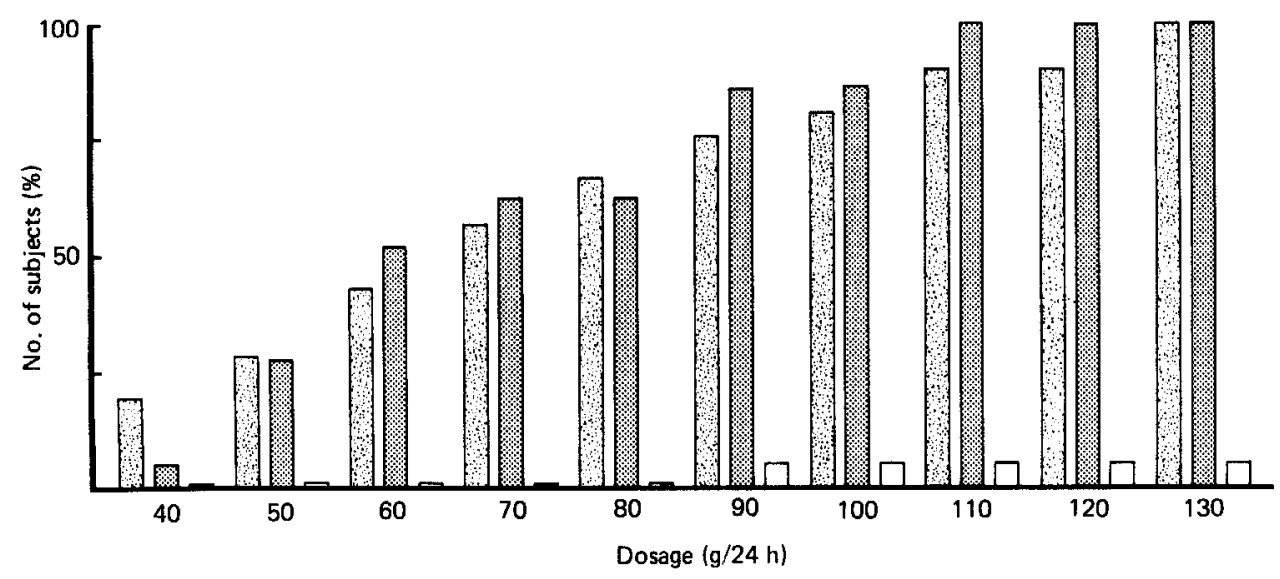

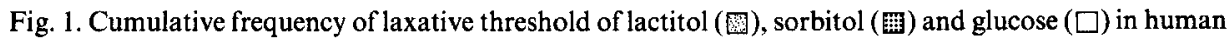
subjects $(n 21)$.

\section{Presentation of results and use of statistics}

The mean laxative threshold for each test substance is presented and in addition the cumulative frequency of laxative threshold for the three test substances are also presented. The laxative threshold and side-effect scores of lactitol, sorbitol and glucose were compared pairwise using Wilcoxon signed-rank test.

\section{Ethical considerations}

This study was approved by the Ethical Committee of the Central Middlesex Hospital. The total body radiation dose from the perfused isotope was estimated to be $<5$ mrad.

\section{RESULTS}

Jejunal perfusion experiments

Galactose could not be detected in portions of the test solutions or intestinal aspirates obtained during perfusion of the four test solutions. As shown in Table 2, there was no significant disappearance of ${ }^{14} \mathrm{C}$ label during the perfusion experiments. 
Table 3. Total symptom score for the last 3 d of each study period for human subjects

(Mean values and standard deviations. The length of each study period was $10 \mathrm{~d}$ if the test substance was tolerated according to protocol design. The study period was shorter if the administration of a test substance was discontinued either because of diarthoea or the development of gastrointestinal symptoms)

\begin{tabular}{|c|c|c|c|c|c|c|}
\hline \multirow[b]{3}{*}{ Symptoms } & \multicolumn{6}{|c|}{ Scores } \\
\hline & \multicolumn{2}{|c|}{ Placebo } & \multicolumn{2}{|c|}{ Lactitol } & \multicolumn{2}{|c|}{ Sorbitol } \\
\hline & Mean & $\mathrm{SD}$ & Mean & $\mathrm{SD}$ & Mean & SD \\
\hline Flatus & $1 \cdot 1$ & 0.5 & $8 \cdot 3$ & $0 \cdot 8$ & $7 \cdot 3$ & $1 \cdot 1$ \\
\hline Abdominal pain & 0.4 & 0.4 & $4 \cdot 2$ & $1 \cdot 1$ & $4 \cdot 3$ & $1 \cdot 1$ \\
\hline Bloating & 0.9 & 0.5 & $5 \cdot 2$ & 0.9 & 50 & $1 \cdot 3$ \\
\hline Borborygmi & $1 \cdot 0$ & 0.6 & $5 \cdot 7$ & $0 \cdot 4$ & 5.9 & $1 \cdot 1$ \\
\hline
\end{tabular}

\section{Laxative threshold study}

All subjects admitted that they could not differentiate the three sugars by their appearance or taste. All subjects were able to take the placebo sugar glucose up to the final dose of $130 \mathrm{~g} / 24 \mathrm{~h}$, although one subject developed mild diarrhoea. Cumulative frequency distribution of laxative threshold of lactitol, sorbitol and placebo are shown in Fig. 1. There was no significant difference in the laxative threshold of lactitol and sorbitol. The mean laxative threshold dose of lactitol was 74 (SE 6) g/24 h. Lactitol administration was discontinued in fifteen subjects on account of diarrhoea and in four subjects because of severe gastrointestinal symptoms. Only two subjects reached the maximum predetermined dose of $130 \mathrm{~g} / 24 \mathrm{~h}$. With regard to sorbitol, the mean laxative threshold dose was 71 (SE 5) g/24 h. Sorbitol administration was discontinued in eleven subjects on account of diarrhoea and in seven subjects because of severe symptoms. Only three subjects reached the maximum predetermined dose of $110 \mathrm{~g} / 24 \mathrm{~h}$.

Although the administration of lactitol and sorbitol was discontinued most often because of diarrhoea, it was common for subjects also to develop significant gastrointestinal symptoms. The scores of each of the symptoms accumulated over the last $3 \mathrm{~d}$ before the discontinuation of each of the study periods are summarized in Table 3 . There was no statistically significant difference in the symptoms produced by lactitol or sorbitol whereas the placebo produced significantly less symptoms $(P<0.001)$.

\section{DISCUSSION}

Lactitol is a disaccharide and consequently has two possible modes of absorption. Either there could be uptake of the intact molecule in the unhydrolysed form or, as is the case for naturally occurring disaccharides sucrose, maltose and lactose, brush-border hydrolysis would precede uptake of its liberated monosaccharides (Gray \& Santiago, 1966; McMichael et al. 1967; Cook, 1973). Aspirates were analysed for free galactose because if significant brush-border hydrolysis of lactitol had occurred during the perfusion experiments, a proportion of the liberated monosaccharides would have diffused back into the gut lumen (Silk \& Dawson, 1979). Galactose could not be detected in any of the intestinal aspirates so it is unlikely that lactitol was hydrolysed by the brush-border hydrolases. There was no significant disappearance of label during our perfusion studies. We therefore conclude that lactitol was not absorbed intact in the unhydrolysed form. This disaccharide is therefore not assimilated by the human small intestine.

Lactitol is very water-soluble, heat-stable and has a mild refreshing taste (Saijonmaa $e t$ 
al. 1978; van Valthuijsen, 1979). The hygroscopicity of lactitol is comparable to that of xylitol and less than that of sorbitol (Saijonmaa et al. 1978). As our findings show that lactitol is not absorbed by the human small intestine, it would appear to have several applications as a substitute for sucrose in foodstuffs (e.g. diabetic and low-energy diets) and, because of its weak hygroscopicity, in various diabetic fine bakery wares and as a coating for moisture-sensitive products.

It should be appreciated, however, that although lactitol is not absorbed in the small intestine, it does not necessarily have a low-energy value. Colonic bacteria, such as Escherichia coli, are capable of metabolizing lactitol (Saijonmaa et al. 1978) and recent studies performed on normal subjects in our laboratory have shown that lactitol ingestion is associated with a fall in the $\mathrm{pH}$ of the right colon (Patil et al. 1985). It seems likely that the cause of fall in $\mathrm{pH}$ is due to liberation of short-chain fatty acids as a consequence of bacterial metabolism. Short-chain fatty acids are a readily utilizable energy source and avidly absorbed from the human colon (McNeil et al. 1978; Ruppin et al. 1980). There is a real need therefore for the energy value of lactitol to be characterized before a final recommendation can be made of the potential role for this sugar in foodstuffs.

The second part of the study was carried out to define tolerance levels and laxative threshold of lactitol because of the fact that an unabsorbed sugar is likely to produce diarrhoea, bloating and abdominal pain. The experimental design was based according to the estimated per capita consumption (if lactitol were to replace sucrose and sorbitol) whereby two or more boluses of lactitol are likely to be ingested each day. The results clearly show that gastrointestinal side effects are likely to limit the use of lactitol at higher doses. Equally though, our findings suggest that it would be quite acceptable to administer up to $40 \mathrm{~g} \mathrm{lactitol} / \mathrm{d}$. Lactitol and sorbitol tolerance as well as laxative threshold are similar. Although outside the scope of the present study, it appears that lactitol at doses in excess of $70 \mathrm{~g} / \mathrm{d}$ acts as a very satisfactory cathartic agent. The related disaccharide lactulose is currently widely used as a cathartic agent. Lactulose, however, has an excessively sweet taste which is unacceptable to a number of patients, many of whom experience nausea after taking it. Lactitol, with its more pleasant taste, may therefore be an ideal successor to lactulose for the treatment of constipation.

The authors are grateful to Express Dairies (UK) Ltd for financial support and encouragement.

\section{REFERENCES}

Cook, G. C. (1973). Clinical Science 44, 425-428.

Gray, G. M. \& Santiago, N. (1966). Gastroenterology 51, 489-498.

Holdsworth, C. D. \& Dawson, A. M. (1964). Clinical Science 27, 371-379.

McMichael, H. B., Webb, J. \& Dawson, A. M. (1967). Clinical Science 33, 135-145.

McNeil, N. I., Cummings, J. H. \& James, W. P. T. (1978). Gut 19, 819-822.

Fatil, D. H., Westaby, D., Mahida, Y. R., Palmer, K. R., Rees, R., Clark, M. L. \& Silk, D. B. A. (1985). Gut 26 , A1125.

Rommel, K., Bernt, E., Schmitz, F. \& Grimmel, K. (1968). Klinische Wochenschrift 46, 936-940.

Ruppin, H., Bar-Meir, S., Soergel, K. H., Wood, C. M. \& Schmitt, M. G. (1980). Gastroenterology 78, 1500-1507.

Saijonmaa, T., Heikonen, M., Kreula, M. \& Linko, P. (1978). Milchwisserschaft 33, 733-736.

Silk, D. B. A. \& Dawson, A. M. (1979). In International Review of Physiology, vol. III, Gastrointestinal Physiology, pp. 151-204 [R. K. Crane, editor]. Baltimore: University Park Press.

Sladen, G. E. \& Dawson, A. M. (1970). Gut 11, 947-954

van Valthuijsen, J. A. (1979). Journal of Agricultural and Food Chemistry 27, 680-686.

Wick, A. N., Alman, M. C. \& Joseph, L. (1951). Journal of American Pharmacology 40, 542-544. 